\title{
EXPLICIT FORMULAS FOR THE COEFFICIENTS OF FABER POLYNOMIALS WITH RESPECT TO UNIVALENT FUNCTIONS OF THE CLASS $\Sigma$
}

\author{
PAVEL G. TODOROV
}

\begin{abstract}
In this note, we obtain three natural explicit formulas for the coefficients of the Faber polynomials with respect to the univalent functions of the class $\Sigma$. As an application, we obtain two new explicit formulas for the Grunsky coefficients of functions in $\Sigma$; these formulas are simpler than those due to Schur [2] and Hummel [4]. The method used in this paper is different from the one that we used to obtain explicit expressions for the Grunsky coefficients for functions of the class $S$ [6].
\end{abstract}

Let $\Sigma$ denote the class of functions

$$
F(z)=z+\sum_{n=0}^{\infty} \alpha_{n} z^{-n}
$$

which are meromorphic and univalent for $|z|>1$, and let

$$
\Phi(t)=t+\sum_{n=0}^{\infty} \beta_{n} t^{-n}
$$

denote the inverse of a function $F$ in $\Sigma$. Let $S$ denote the class of functions

$$
f(z)=\sum_{n=1}^{\infty} a_{n} z^{n}, \quad a_{1}=1,
$$

which are analytic and univalent for $|z|<1$, i.e.,

$$
F(z)=\frac{1}{f(1 / z)} \in \Sigma, \quad|z|>1 .
$$

It follows from results due to Grunsky [1], Schur [2] and Schiffer [3] that the Faber polynomials $\Phi_{m}(t)$ with respect to $F(z)$ are the coefficients in the expansion

$$
\log \frac{F(z)-t}{z}=-\sum_{m=1}^{\infty} \frac{1}{m} \Phi_{m}(t) z^{-m}
$$

as well as the regular part of the Laurent expansion of $[\Phi(t)]^{m}$. The coefficients in the expansions

$$
\Phi_{m}[F(\zeta)]=\zeta^{m}+\sum_{n=1}^{\infty} c_{m n} \zeta^{-n}
$$

Received by the editors January 29, 1980 and, in revised form, May 28, 1980 and August 8, 1980.

1980 Mathematics Subject Classification. Primary 30C45, 30C50; Secondary 30C30.

Key words and phrases. Faber polynomial, Grunsky coefficient, Faà di Bruno formula for the $n$th derivative of composite functions. 
and

$$
\log \frac{z-\zeta}{F(z)-F(\zeta)}=\sum_{m, n=1}^{\infty} g_{m n} z^{-m \zeta^{-n}}
$$

are connected by the relations

$$
c_{m n}=m g_{m n}, \quad m, n=1,2, \ldots,
$$

where the $g_{m n}$ are the Grunsky coefficients of $F(z)$. In (7), and elsewhere in this paper, we use only the principal values of the logarithms and powers that appear in the formulas.

Explicit formulas for the coefficients $c_{m n}$ and $g_{m n}$ in terms of the $\alpha_{n}$ in (1) and the $a_{n}$ in (3) were found by Schur [2] and Hummel [4], respectively. By methods we have used elsewhere [5], [6] it is possible to obtain additional explicit expressions for the coefficients $g_{m n}$ in terms of the coefficients $a_{n}$ in (3). Despite these small successes, the problem of finding explicit expressions for the coefficients of the Faber polynomials $\Phi_{m}(t)$ themselves in terms of the coefficients in (1), (2), and (3) has remained open.

In this paper, we solve that problem; we find explicit formulas for the coefficients of the Faber polynomials in terms of the $\alpha_{n}, \beta_{n}$ and $a_{n}$. As an application we obtain, in a natural way, three explicit formulas for the coefficients in (6) in terms of the coefficients $\alpha_{n}, \alpha_{n}$ and $\beta_{n}$, and $a_{n}$, in turn. In particular, we obtain two new explicit formulas for the Grunsky coefficients $g_{m n}$ in terms of $\alpha_{n}$, and $\alpha_{n}$ and $\beta_{n}$, respectively.

We shall make use of the factorial polynomials

$$
(x)_{k}=x(x-1)(x-2) \cdots(x-k+1), \quad k=1,2, \ldots,(x)_{0}=1 .
$$

For arbitrary $c_{1}, c_{2}, \ldots, c_{n}$ we shall also use the homogeneous isobaric polynomials of degree $k$ and weight $n$ introduced elsewhere [5], [6]:

$$
\begin{gathered}
C_{n, k}\left(c_{1}, \ldots, c_{n-k+1}\right)=\sum \frac{\left(c_{1}\right)^{\nu_{1}} \cdots\left(c_{n-k+1}\right)^{n_{n-k+1}}}{\nu_{1} ! \cdots \nu_{n-k+1} !}, \quad 1<k<n, \\
C_{n, 0}\left(c_{1}, \ldots, c_{n+1}\right)=0, \quad C_{0,0}\left(c_{1}\right)=1,
\end{gathered}
$$

where the sum is taken over all nonnegative integers $\nu_{1}, \ldots, \nu_{n-k+1}$ satisfying $\nu_{1}+\cdots+\nu_{n-k+1}=k, \nu_{1}+2 \nu_{2}+\cdots+(n-k+1) \nu_{n-k+1}=n$. The polynomials (10) are easily computed if one uses the recursion relations

$$
\begin{aligned}
C_{n, k} & =\frac{1}{k} \sum_{\mu=1}^{n-k+1} c_{\mu} C_{n-\mu, k-1}, \quad 1<k<n, 1<n, \\
C_{n, k} & \equiv C_{n, k}\left(c_{1}, \ldots, c_{n-k+1}\right), \\
C_{n, 0} & =0, \quad C_{0,0}=1, \quad C_{n, 1}=c_{n}, \quad C_{n, n}=c_{1}^{n} / n ! .
\end{aligned}
$$

We note that the expression $k ! C_{n, k}\left(c_{1}, \ldots, c_{n-k+1}\right)$ is the coefficient $C_{z^{n}}$ of $z^{n}$ in the $k$ th power of the formal power series $\left(c_{1} z+\ldots\right)$, that is,

$$
C_{z^{n}}\left(\sum_{m=1}^{\infty} c_{m} z^{m}\right)^{k} \equiv k ! C_{n, k}\left(c_{1}, \ldots, c_{n-k+1}\right), \quad 1<k<n .
$$

(See (24), below, and/or formula (45) in [6].) 
We now proceed to establish our results, starting with an expression for the coefficients of the Faber polynomials $\Phi_{m}(t)$ in terms of the $\alpha_{n}$ in (1).

THEOREM 1. In terms of the coefficients $\alpha_{n}$ in (1), the Faber polynomials $\Phi_{m}(t)$ are given by

$$
\Phi_{m}(t)=-m \Psi_{m}+m \sum_{n=1}^{m} \frac{1}{n} \Psi_{m-n}(n) t^{n}, \quad m=1,2, \ldots
$$

where

$$
\Psi_{m} \equiv C_{z^{-m}}\left[\log \frac{F(z)}{z}\right]=\sum_{k=1}^{m}(-1)^{k-1}(k-1) ! C_{m, k}\left(\alpha_{0}, \alpha_{1}, \ldots, \alpha_{m-k}\right),
$$

and where

$$
\Psi_{m-n}(n) \equiv C_{z^{-m+n}}\left[\frac{F(z)}{z}\right]^{-n}=\sum_{k=0}^{m-n}(-n)_{k} C_{m-n, k}\left(\alpha_{0}, \alpha_{1}, \ldots, \alpha_{m-n-k}\right) .
$$

Proof. If we replace $z$ by $1 / z$ in (5), and if we use $\Psi(z) \equiv z F(1 / z)=1+$ $\sum_{n=1}^{\infty} \alpha_{n-1} z^{n},|z|<1$, then we obtain

$$
\log (\Psi(z)-t z)=\log \Psi(z)+\log \left(1-\frac{t z}{\Psi(z)}\right)=-\sum_{m=1}^{\infty} \frac{1}{m} \Phi_{m}(t) z^{m}
$$

If we apply the Faà di Bruno "precise formula" for the $n$th derivative of composite functions, developed in an earlier publication [5, Theorem 1], to the composite function $\log \Psi(z)=(\log w) \circ \Psi(z)$, then we obtain

$$
\begin{gathered}
\log \Psi(z)=\sum_{m=1}^{\infty} \Psi_{m} z^{m}, \\
\Psi_{m} \equiv C_{z m}[\log \Psi(z)]=\sum_{k=1}^{m}(-1)^{k-1}(k-1) ! C_{m, k}\left(\alpha_{0}, \ldots, \alpha_{m-k}\right) .
\end{gathered}
$$

Since we also have the expansion,

$$
\log \left(1-\frac{t z}{\Psi(z)}\right)=-\sum_{n=1}^{\infty} \frac{1}{n} t^{n} z^{n}[\Psi(z)]^{-n},
$$

another application of the Faà di Bruno formula, this time to the composite function on $[\Psi(z)]^{-n}=w^{-n} \circ \Psi(z)$, yields

$$
[\Psi(z)]^{-n}=1+\sum_{m=1}^{\infty} \Psi_{m}(n) z^{m}
$$

where, making use of (9)

$$
\begin{gathered}
\Psi_{m}(n) \equiv C_{z^{m}}[\Psi(z)]^{-n}=\sum_{k=1}^{m}(-n)_{k} C_{m, k}\left(\alpha_{0}, \ldots, \alpha_{m-k}\right), \\
\Psi_{0}(n)=1, \quad n=1,2, \ldots .
\end{gathered}
$$

If we now use (16) and (17), we obtain

$$
\begin{aligned}
\log \left(1-\frac{t z}{\Psi(z)}\right) & =-\sum_{n=1}^{\infty} \frac{1}{n} t^{n} \sum_{m=n}^{\infty} \Psi_{m-n}(n) z^{m} \\
& =-\sum_{m=1}^{\infty} z^{m} \sum_{n=1}^{m} \frac{1}{n} \Psi_{m-n}(n) t^{n}
\end{aligned}
$$


Finally, from (14), (15) and (19) we deduce

$$
\log (\Psi(z)-t z)=-\sum_{m=1}^{\infty} z^{m}\left[-\Psi_{m}+\sum_{n=1}^{m} \frac{1}{n} \Psi_{m-n}(n) t^{n}\right] .
$$

Now the expansions in (15), (19) and (20) converge for all sufficiently small $|t|$ and/or for all $z$ close enough to the origin so that $\Psi(z) \neq 0$ there. If we now compare the coefficients of (14) and (20), then we obtain (11), (12) and (13). This completes our proof of Theorem 1.

Now we find expressions for the coefficients of the polynomials $\Phi_{m}(t)$ in terms of the coefficients $\beta_{n}$ in (2).

THEOREM 2. In terms of the $\beta_{n}$ in (2), the Faber polynomials $\Phi_{m}(t)$ are given by the formulas

where

$$
\Phi_{m}(t)=\sum_{n=0}^{m} \phi_{2 m-n}(m) t^{n}, \quad m=1,2, \ldots
$$

$$
\begin{aligned}
\phi_{2 m-n}(m) & \equiv C_{t^{-2 m+n}}\left[\frac{\Phi(t)}{t^{2}}\right]^{m} \\
& =m ! C_{2 m-n, m}\left(\beta_{-1}, \beta_{0}, \ldots, \beta_{m-n-1}\right), \quad \beta_{-1}=1 .
\end{aligned}
$$

Proof. If we replace $t$ by $1 / t$ in (2), then we obtain a series

$$
\phi(t) \equiv t^{2} \Phi\left(\frac{1}{t}\right)=\sum_{n=1}^{\infty} \beta_{n-2} t^{n}, \quad \beta_{-1}=1,
$$

that converges in the largest disc with center at $t=0$ not containing a singular point of $\phi(t)$. The Faà di Bruno "precise formula", now applied to the composite function $[\phi(t)]^{m}=w^{m} \circ \phi(t)$, yields a series

$$
\begin{gathered}
{[\phi(t)]^{m}=\sum_{n=m}^{\infty} \phi_{n}(m) t^{n},} \\
\phi_{n}(m) \equiv C_{t^{n}}[\phi(t)]^{m}=m ! C_{n, m}\left(\beta_{-1}, \beta_{0}, \ldots, \beta_{n-m-1}\right), \quad \beta_{-1}=1,
\end{gathered}
$$

that has the same radius of convergence as the series for $\phi(t)$. From (23) and (24) we obtain the Laurent expansion

$$
[\Phi(t)]^{m}=\sum_{n=0}^{m} \phi_{2 m-n}(m) t^{n}+\sum_{n=1}^{\infty} \phi_{2 m+n}(m) t^{-n} .
$$

From (25) we obtain the series (21) whose coefficients are given by (22). This completes our proof of Theorem 2.

We now find the coefficients of $\Phi_{m}(t)$ in terms of the $a_{n}$ in (3).

THEOREM 3. The Faber polynomials $\Phi_{m}(t)$ have the form

$$
\begin{gathered}
\Phi_{m}(t)=m b_{m}+m \sum_{k=1}^{m}(k-1) ! C_{m, k}\left(a_{1}, \ldots, a_{m-k+1}\right) t^{k}, \quad m=1,2, \ldots, \\
b_{m} \equiv C_{z^{m}}\left[\log \frac{f(z)}{z}\right]=\sum_{k=1}^{m}(-1)^{k-1}(k-1) ! C_{m, k}\left(a_{2}, \ldots, a_{m-k+2}\right) \\
m=1,2, \ldots, \quad
\end{gathered}
$$

and where the $a_{n}$ are those in (3) above. 
Proof. If we replace $z$ by $1 / z$ in $F(z)$ in (4) and (5), we obtain

$$
\log z\left(\frac{1}{f(z)}-t\right)=\log (1-t f(z))-\log \frac{f(z)}{z}=-\sum_{m=1}^{\infty} \frac{1}{m} \Phi_{m}(t) z^{m} .
$$

If we now apply the Faà di Bruno formula once again, this time to the composite function $\log [f(z) / z]=[\log w] \circ[f(z) / z]$, then we obtain

$$
\log \frac{f(z)}{z}=\sum_{m=1}^{\infty} b_{m} z^{m}, \quad|z|<1,
$$

where the $b_{m}$ are those in (26). Another application of the Faà di Bruno formula, to $\log (1-t f(z))=\log (1-t w) \circ f(z)$, yields

$$
\begin{gathered}
\log (1-t f(z))=-\sum_{m=1}^{\infty} c_{m}(t) z^{m} \\
c_{m}(t)=\sum_{k=1}^{m}(k-1) ! C_{m, k}\left(a_{1}, \ldots, a_{m-k+1}\right) t^{k}, \quad a_{1}=1 .
\end{gathered}
$$

The series in (29) converges for sufficiently small $|t|$ and $|z|$.

From (27), (28) and (29) we obtain

$$
\log \left[z\left(\frac{1}{f(z)}-t\right)\right]=-\sum_{m=1}^{\infty}\left[b_{m}+c_{m}(t)\right] z^{m}
$$

We now compare the expansions in (27) and (30), to obtain (26); this completes our proof.

We remark that for $F(z)$ given by (4), a comparison of the expansions (11), (21) and (26) would yield additional identities analogous to those obtained by comparing (11) and (21).

We turn now to the task of obtaining explicit forms for the coefficients of the expansion in (6) corresponding to each of the three cases we have just considered, and those forms in turn will yield three explicit expressions for the Grunsky coefficients $g_{m n}$ in (7).

THEOREM 4. In terms of the coefficients $\alpha_{n}$ in (1), the Grunsky coefficients $g_{m n}$ in (7) are given by

$$
\begin{aligned}
g_{m n}= & \sum_{r=1}^{m} \sum_{k=0}^{m-r}(-1)^{k}(r+k-1) ! C_{2 r+n, r}\left(\alpha_{-1}, \alpha_{0}, \ldots, \alpha_{r+n-1}\right) \\
& \cdot C_{m-r, k}\left(\alpha_{0}, \alpha_{1}, \ldots, \alpha_{m-r-k}\right),
\end{aligned}
$$

where $\alpha_{-1}=1$.

Proof. With $t=F(\zeta),|\zeta|>1$, in (11) we obtain

$$
\Phi_{m}(F(\zeta))=-m \Psi_{m}+m \sum_{r=1}^{m} \frac{1}{r} \Psi_{m-r}(r)[F(\zeta)]^{r},
$$

and then another application of the Faà di Bruno formula, as in the proof of 
Theorem 2, yields

$$
\begin{aligned}
& {[F(\zeta)]^{r}=\sum_{n=0}^{r} h_{2 r-n}(r) \zeta^{n}+\sum_{n=1}^{\infty} h_{2 r+n}(r) \zeta^{-n}, \quad|\zeta|>1,} \\
& h_{2 r \mp n}(r) \equiv C_{\zeta^{-2 r \pm n}}\left[\frac{F(\zeta)}{\zeta^{2}}\right]^{r}=r ! C_{2 r \mp n, r}\left(\alpha_{-1}, \alpha_{0}, \ldots, \alpha_{r \mp n-1}\right), \quad \alpha_{-1}=1 .
\end{aligned}
$$

In (33), the upper (lower) signs correspond to one another. Now from (32), and (33) we obtain

$$
\begin{aligned}
\Phi_{m}(F(\zeta))= & m\left[-\Psi_{m}+\sum_{r=1}^{m} \frac{1}{r} \Psi_{m-r}(r) h_{2 r}(r)\right] \\
& +m \sum_{n=1}^{m} \zeta^{n} \sum_{r=n}^{m} \frac{1}{r} \Psi_{m-r}(r) h_{2 r-n}(r) \\
& +m \sum_{n=1}^{\infty} \zeta^{-n} \sum_{r=1}^{m} \frac{1}{r} \Psi_{m-r}(r) h_{2 r+n}(r) .
\end{aligned}
$$

Since the expansions in (6) and (34) must be the same, it follows that

$$
\begin{aligned}
\sum_{r=1}^{m} \frac{1}{r} \Psi_{m-r}(r) h_{2 r}(r) & =\Psi_{m}, \quad m=1,2, \ldots, \\
\sum_{r=n}^{m} \frac{1}{r} \Psi_{m-r}(r) h_{2 r-n}(r) & =0, \quad 1<n<m-1,2<m,
\end{aligned}
$$

must hold, and hence the coefficients $c_{m n}$ in (6) satisfy the relations

$$
c_{m n}=m \sum_{r=1}^{m} \frac{1}{r} h_{2 r+n}(r) \Psi_{m-r}(r) \text {. }
$$

From (8) and (35), keeping (13) and (33) in mind, we obtain (31). This completes our proof of Theorem 4.

We note that our formulas (35), and its equivalent (31), are simpler than corresponding results due to Schur [2, pp. 36-37, formulas (11) and (18)].

THEOREM 5. The Grunsky coefficients $g_{m n}$ in (7) are given by

$$
\begin{aligned}
g_{m n}= & (m-1) ! \sum_{r=1}^{m} r ! C_{2 r+n, r}\left(\alpha_{-1}, \alpha_{0}, \ldots, \alpha_{r+n-1}\right) \\
& \cdot C_{2 m-r, m}\left(\beta_{-1}, \beta_{0}, \ldots, \beta_{m-r-1}\right), \quad \alpha_{-1}=\beta_{-1}=1,
\end{aligned}
$$

where the $\alpha_{n}$ and the $\beta_{n}$ are those in (1) and (2), respectively.

Proof. With $t=F(\zeta),|\zeta|>1$ in (21) and using (33) we have

$$
\Phi_{m}(F(\zeta))=\sum_{n=0}^{m} \zeta^{n} \sum_{r=n}^{m} \phi_{2 m-r}(m) h_{2 r-n}(r)+\sum_{n=1}^{\infty} \zeta^{-n} \sum_{r=1}^{m} \phi_{2 m-r}(m) h_{2 r+n}(r) \text {. }
$$

By comparing (6) and (37) we see that we must have

$$
\sum_{r=n}^{m} \phi_{2 m-r}(m) h_{2 r-n}(r)=0, \quad 0<n<m-1,1<m,
$$


and hence the coefficients $c_{m n}$ in (6) satisfy

$$
c_{m n}=\sum_{r=1}^{m} h_{2 r+n}(r) \phi_{2 m-r}(m) \text {. }
$$

From (8), (22), (33) and (38) we obtain (36). This completes our proof.

It should be noted that (36), in spite of its "simplicity", is a "first" in the sense that it expresses the Grunsky coefficients in explicit form simultaneously in terms of the coefficients of both the function (1) and its inverse (2).

THEOREM 6. In terms of the $a_{n}$ in (3), the Grunsky coefficients $g_{m n}$ in (7) are given by the following explicit formula:

$$
\begin{gathered}
g_{m n}=\sum_{k=1}^{m} \sum_{s=1}^{n+k}(-1)^{s}(k+s-1) ! C_{m, k}\left(a_{1}, \ldots, a_{m-k+1}\right) \\
\cdot C_{n+k, s}\left(a_{2}, \ldots, a_{n+k-s+2}\right), \quad a_{1}=1 .
\end{gathered}
$$

Proof. If we set $t=\left[1 / f\left(\zeta^{-1}\right)\right],|\zeta|>1$, in (26) we obtain

$$
\Phi_{m}\left[\frac{1}{f\left(\zeta^{-1}\right)}\right]=m b_{m}+m \sum_{k=1}^{m}(k-1) ! C_{m, k}\left(a_{1}, \ldots, a_{m-k+1}\right)\left[f\left(\zeta^{-1}\right)\right]^{-k} \text {. }
$$

If we now use the same technique used above in obtaining (17) and (18) we obtain

$$
\begin{aligned}
{\left[\frac{f(z)}{z}\right]^{-k} } & =\sum_{n=0}^{\infty} g_{n}(-k) z^{n}, \quad|z|<1, \\
g_{n}(-k) & =\sum_{s=0}^{n}(-k)_{s} C_{n, s}\left(a_{2}, \ldots, a_{n-s+2}\right) .
\end{aligned}
$$

If we set $z=1 / \zeta,|\zeta|>1$ in (41), then we obtain

$$
\left[f\left(\zeta^{-1}\right)\right]^{-k}=\sum_{n=0}^{k} g_{k-n}(-k) \zeta^{n}+\sum_{n=1}^{\infty} g_{k+n}(-k) \zeta^{-n}
$$

which, in view of (40), yields

$$
\begin{aligned}
\Phi_{m}\left[1 / f\left(\zeta^{-1}\right)\right]= & m\left[b_{m}+\sum_{k=1}^{m}(k-1) ! C_{m, k}\left(a_{1}, \ldots, a_{m-k+1}\right) g_{k}(-k)\right] \\
+ & m\left[\sum_{n=1}^{m} \zeta^{n} \sum_{k=n}^{m}(k-1) ! C_{m, k}\left(a_{1}, \ldots, a_{m-k+1}\right) g_{k-n}(-k)\right. \\
& \left.+\sum_{n=1}^{\infty} \zeta^{-n} \sum_{k=1}^{m}(k-1) ! C_{m, k}\left(a_{1}, \ldots, a_{m-k+1}\right) g_{k+n}(-k)\right] .
\end{aligned}
$$

If we compare (6) with (42), with $F(\zeta) \equiv\left[1 / f\left(\zeta^{-1}\right)\right]$, then we obtain

$$
\begin{gathered}
\sum_{k=1}^{m}(k-1) ! C_{m, k}\left(a_{1}, \ldots, a_{m-k+1}\right) g_{k}(-k)=-b_{m}, \quad m=1,2, \ldots, \\
\sum_{k=n}^{m}(k-1) ! C_{m, k}\left(a_{1}, \ldots, a_{m-k+1}\right) g_{k-n}(-k)=0, \\
1<n<m-1, m=2,3, \ldots, \\
c_{m n}=m \sum_{k=1}^{m}(k-1) ! C_{m, k}\left(a_{1}, \ldots, a_{m-k+1}\right) g_{k+n}(-k), \quad a_{1}=1,
\end{gathered}
$$

which combine with (8) and (41) to give us (39). This completes our proof. 
The explicit formulas (39) for the Grunsky coefficients (indeed, for $p$-symmetric functions) are not new. They have been obtained by a method different from the one used here [6].

\section{REFERENCES}

1. H. Grunsky, Koeffizientenbedingungen für schlicht abbildende meromorphe Funktionen, Math Z. 45 (1939), 29-61.

2. I. Schur, On Faber polynomials, Amer. J. Math. 67 (1945), 33-41.

3. M. Schiffer, Faber polynomials in the theory of univalent functions, Bull. Amer. Math. Soc. 54 (1948), 503-517.

4. J. A. Hummel, The Grunsky coefficients of a schlicht function, Proc. Amer. Math. Soc. 15 (1964), 142-150.

5. P. G. Todorov, New explicit formulas for the coefficients of p-symmetric functions, Proc. Amer. Math. Soc. 77 (1979), 81-86.

6. __ New explicit formulas for the Grunsky coefficients of univalent functions (submitted).

Department of Mathematics, Paissi Hilendarski University, 4000 Plovdiv, Bulgaria

Current address: 20 Lenin Avenue, 4002 Plovdiv, Bulgaria 